\title{
A Computer Simulation of the Nitinol Thermal Expansion under Fast Varying Working Conditions
}

\author{
P. Castelo Ferreira ${ }^{1, a}$, P. Pascoal-Faria ${ }^{1,2, b}$, P. Carreira ${ }^{1, c}$ and N. Alves ${ }^{1,3, d}$ \\ ${ }^{1}$ Centre for Rapid and Sustainable Product Development, Polytechnic Institute of Leiria \\ Instituto Politécnico de Leiria, Rua de Portugal - Zona Industrial, 2430-028 Marinha Grande, \\ Portugal
}

${ }^{2}$ Department of Mathematics, School of Technology and Management, Polytechnic Institute of Leiria Rua General Norton de Matos, Apartado 4133, 2411-901 Leiria, Portugal

${ }^{3}$ Department of Mechanical Engineering, School of Technology and Management, Polytechnic Institute of Leiria

Rua General Norton de Matos, Apartado 4133, 2411-901 Leiria, Portugal

apedro.castelo.ferreira@gmail.com, bpaula.faria@ipleiria.pt, cpedro.s.carreira@ipleiria.pt, dnuno.alves@ipleiria.pt

Keywords: Nitinol, Thermal Expansion Coefficient, Simulation.

\begin{abstract}
We discuss the setup of a simulation on ANSYS to predict the thermal expansion of parts made of Nitinol. A simulation is justified for working conditions in which the part heating is not homogeneous originating a temperature gradient across the part section such that an analytical estimate for the part expansion cannot be calculated. We apply the simulation to the topological optimization of a square section geometry and a bullet geometry for water assisted injection molding. For the topological optimization we consider as parameter the wall thickness and consider both the cases of fast varying temperature and fast varying temperature and pressure.
\end{abstract}

\section{Introduction}

Nitinol is a Nickel Titanium Alloy (NiTi) that was originally manufactured at the Naval Ordnance Laboratory [1]. It exhibits both shape memory effect and superelasticity as well as being very resistance to corrosion and fatigue. Due to the molecular organization of the metal alloy it has two distinct phases, Martensitic phase $(M)$ at lower temperatures and Austenitic phase $(A)$ at higher temperatures. The phase transition $M \rightarrow A$ (rising temperature) takes place in between the start temperature $A_{s}$ and finish temperature $A_{f}>A_{s}$ and the phase transition $A \rightarrow M$ (lowering temperature) takes place between the start temperature $M_{s}$ and the finish temperature $M_{f}<M_{s}$ such that $A_{s}>M_{f}$ and $A_{f}>M_{s}$. The stress-free Martensitic phase (temperature dependent) is characterized by a twinned multi-variant crystallography structure, in addition there is a stress induced Martensitic phase ( $S$, stress dependent) characterized by a detwinned configuration with a single variant crystallographic structure.

The shape memory effect consists in deforming the material below the temperature $M_{s}$ ( $M$ phase), when it is heated above the temperature $A_{s}$ ( $A$ phase) it recovers its original (recorded) shape. As for superelasticity allows for about 15 times the elastic motion of steel and consists in that, when the material is above the temperature $A_{s}$, under mechanical loads the stress induces a phase transition $A \rightarrow S$, once the load is removed the phase transition $S \rightarrow A$ also restores its original (recorded) phase. Hence both for the thermal and stress induced phase transitions the material response shows a hysteresis cycle.

Although originally the study of NiTi was carried mostly for the 1:1 Nickel weight percentage of $55 \%$ wt corresponding to the atomic percentage of $49.9 \%$, soon it was realized that the specific values of the transition temperatures and mechanical properties highly depend on the Nickel percentage [2] (see references therein), specifically for NiTi alloys the Nickel atomic percentage ranges from 
$49.0 \%$ a to $50.7 \%$ a corresponding to $M_{s}$ between $+100^{\circ} \mathrm{C}$ to $-50^{\circ} \mathrm{C}$ (respectively) and the ductility is reduced as Nickel percentage is increased. As for the addition of other elements to the alloy, Platinum and Palladium allow to change $M_{s}$ by $-10^{\circ} \mathrm{C}$ to $+250^{\circ} \mathrm{C}$, Niobium allows to increased the hysteresis width and Copper allows to reduce the hysteresis width. Also both the transition temperatures and mechanical properties of the alloys are highly dependent on the manufacturing and treatment of the parts $[3,4,5,6]$, in particular it is claimed that for lower Nickel percentages the cold working is the predominant factor in setting the material properties while for higher Nickel percentages the heating treatment is the predominant factor. Although industry standards exist [7], it is still hard to fully compare existing results and discussions in the literature as the specific alloy percentages, part manufacturing process and treatment are often omitted.

Table 1: Shape memory effect parameters considered in the simulation with reference temperature matching $M_{f}$.

\begin{tabular}{ll|c}
\hline Hardening Parameter $(h)$ & {$[P a]$} & $5.25 \times 10^{8}$ \\
Elastic Limit $(R)$ & {$[P a]$} & $8.44 \times 10^{7}$ \\
Temperature Scaling Parameter $(\beta)$ & {$\left[\mathrm{Pa}^{\circ} C^{-1}\right]$} & $3.64 \times 10^{6}$ \\
Maximum Transformation Strain $\left(\epsilon_{L}\right)$ & & 0.07 \\
Martensite Modulus $\left(E_{m}\right)$ & {$[P a]$} & $6.895 \times 10^{10}$ \\
Lode Transformation Parameter $(m)$ & & 0.0875 \\
\hline
\end{tabular}

In this work our main objective is to analyze how NiTi part dimensions vary with temperature. In particular, for a given NiTi part, we study how the dimensions at ambient temperature are related with the dimensions at working temperature and pressure. We note that for relatively long operation times, homogeneous temperature and pressure can be assume. Hence the steady state approximation is valid and it is possible to analytically compute the part dimensions at working conditions. However for fast varying working temperatures and pressures we have to account for thermal conduction and non homogeneous expansions. For that purpose we are going to setup a simulation in ANSYS. Reciprocally the simulation also allows to relate dimensions at manufacturing temperature to dimensions at ambient temperature, not exclusively but also including, digital directly manufactured parts.

\section{Simulation Setup}

In addition to the NiTi properties dependence on the alloy composition and part manufacturing and treatment, we note that generally the material properties are distinct in both Martensitic and Austenitic phases. Of relevance to the present study we note that the Coefficient of Thermal Expansion (CTE) has been measured experimentally for distinct samples with distinct treatments $[3,5]$ and generally varies in between two distinct stable values for both NiTi phases. Moreover the CTE during the phase transition is generally non-linear and as expected due to the shape memory effect gives rise to a thermal hysteresis cycle [3].

The simulation of the shape memory effect and superelasticity is today available in commercial softwares, for the particular case of simulation using the software ANSYS see for instance [8]. To setup a simulation with specific NiTi material properties including the shape memory effect let us consider the following phase transition temperatures

$$
M_{f}=40\left[{ }^{\circ} \mathrm{C}\right], M_{s}=50\left[{ }^{\circ} \mathrm{C}\right], A_{s}=95\left[{ }^{\circ} \mathrm{C}\right], A_{f}=105\left[{ }^{\circ} \mathrm{C}\right] .
$$

There is already a pre-defined material for shape memory effect, for which we consider the parameters listed in table 1 with reference temperature matching $M_{f}$.

The specific model employed is defined an discussed in ANSYS documentation [9]. The plasticity and material dilatation for the shape memory effect are modeled based on the stress matrix. The 
Temperature Scaling Parameter $\beta$ controls the phase transition through the Maxwell stress describing the dependence on the temperature of the shift of the stress hysteresis cycle. However it does not describe neither the standard plasticity nor the standard thermal expansion of the material which must be supplemented independently of the shape memory effect. For the simulation we consider the material properties listed in table 2 for the Martensitic and Austenitic phases.

Table 2: NiTi Mechanical and Thermal Properties of NiTi for the Martensitic phase and the Austenitic phase considered in the simulation.

\begin{tabular}{ll|c|c} 
& & Martensitic & Austenitic \\
\hline \hline Density & {$\left[\mathrm{kg} \mathrm{m}^{-3}\right]$} & 6500 & 6500 \\
Coefficient of Thermal Expansion & {$\left[{ }^{\circ} \mathrm{C}^{-1}\right]$} & $6.6 \times 10^{-6}$ & $9.4 \times 10^{-6}$ \\
Young's Modulus & {$[\mathrm{Pa}]$} & $4 \times 10^{10}$ & $8 \times 10^{10}$ \\
Yield Strength & {$[\mathrm{Pa}]$} & $1.05 \times 10^{8}$ & $4.5 \times 10^{8}$ \\
Tensile Ultimate Strength & {$[\mathrm{Pa}]$} & $1.2 \times 10^{9}$ & $1.07 \times 10^{9}$ \\
\hline Thermal Conductivity & {$\left[\mathrm{W} \mathrm{m} \mathrm{m}^{\left.-1{ }^{o} \mathrm{C}^{-1}\right]}\right.$} & 8.6 & 18 \\
Specific Heat & {$\left[\mathrm{cal} \mathrm{g}^{\left.-1{ }^{o} \mathrm{C}^{-1}\right]}\right.$} & 0.2 & 0.2 \\
\hline
\end{tabular}

To fully reproduce the thermal hysteresis cycle we consider as approximation a linear interpolation between the values of each of the material properties for both temperatures $A_{s}$ and $A_{f}$ for rising temperatures $(M \rightarrow A)$ and $M_{s}$ and $M_{f}$ for lowering temperatures $(A \rightarrow M)$. In particular, for both rising and lowering temperature branches of the hysteresis cycle, the material expansion is modeled by the Isotropic Secant Coefficient of Thermal Expansion (see [9]) with the same Zero-Thermal-Strain Reference Temperature $T_{\text {ref }}$ for both directions of the cycle which ensures that below $M_{f}$ and above $A_{f}$ the modeled material expansion is exactly matched independently of the temperature gradient direction. For the simulation we consider the reference temperature $T_{\text {ref }}$ to match the environment temperature $T_{0}$

$$
T_{\text {ref }}=T_{0}=22^{\circ} C \text {. }
$$

To test the setup we consider a simple geometry corresponding to a bar of square section $4 \mathrm{~mm} \times$ $4 \mathrm{~mm}$ (aligned with the $y z$-plane) and length of $l_{0}=10 \mathrm{~mm}$ (aligned with the $x$-axis). The geometry dimensions are quoted for the environment temperature. We simulate only $1 / 4$ of the bar such that the point at $(0,0,0) \mathrm{mm}$ is fixed. For the evaluation of displacements and strain we consider the point along the $x$ bar symmetry axis at $x=l=10 \mathrm{~mm},(0,0,10) \mathrm{mm}$. For the simulation setup see figure 1 .
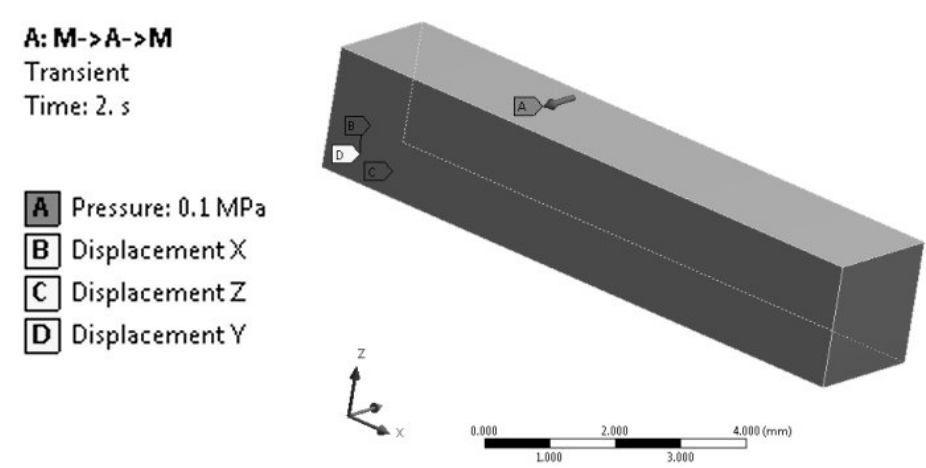

Fig. 1: Simulation setup for $1 / 4$ of a NiTi bar with dimensions $4 \mathrm{~mm} \times 4 \mathrm{~mm} \times 10 \mathrm{~mm}$. The bar axis is align with the $x$ axis for $y=z=0$. 
Table 3: Analytical and simulated values for the expansion along the $x$-axis and $y, z$-axis for $T=$ $150^{\circ} \mathrm{C}$.

\begin{tabular}{ll|c|c}
\multicolumn{2}{|c|}{ axis } & analitical & simulated \\
\hline \hline$x$ & {$[\mathrm{~mm}]$} & 0.0120320 & $0.0120300 \pm 0.0000120$ \\
$y, z$ & {$[\mathrm{~mm}]$} & 0.00240640 & $0.00240610 \pm 0.00000241$ \\
\hline
\end{tabular}

We recall that the coefficient of thermal expansion is defined with respect to the original length at environment temperature $T_{\text {ref }}$ (it corresponds to the secant for each temperature), such that for experimentally measured values at each of the NiTi phases for the coefficient

$$
\alpha(T)=\frac{\Delta l}{l_{0}} \frac{1}{T-T_{\mathrm{ref}}},
$$

the material expansion can be analytically computed $\Delta l=l_{0} \alpha\left(T-T_{0}\right)$. For a given part under a known pressure and thermal distribution, both to test the simulation and evaluate the effective value of $\alpha$ at each time step $i$ we obtain the discretized values of the coefficient of thermal expansion to be

$$
\alpha_{i}=\frac{\Delta l_{i}}{l_{0}} \frac{1}{T_{i}-T_{\text {ref }}} .
$$

To test the simulation we consider that the bar is subject to a homogeneous pressure of $0.1 \mathrm{MPa}$ and impose a homogeneous temperature profile up to the time of $2 \mathrm{~s}$ rising from $T(t=0.1 \mathrm{~s})=22^{\circ} \mathrm{C}$ to $T(t=1 s)=150^{\circ} \mathrm{C}$ and dropping again to $T(t=1.9 \mathrm{~s})=22^{\circ} \mathrm{C}$.

We run the simulation setting the convergence criteria for deformations to $0.1 \%$. At the point $(10 \mathrm{~mm}, 2 \mathrm{~mm}, 2 \mathrm{~mm})$ the pressure has the compressive effect $\Delta_{x, P(\mathrm{sim})}=-5 \times 10^{-6} \pm 5 \times 10^{-9} \mathrm{~mm}$, $\Delta_{y, P}=\Delta_{z, P(\mathrm{sim})}=-10^{-6} \pm 10^{-9} \mathrm{~mm}$. Within the error bars for the simulation the thermal expansion at $T(t=1 \mathrm{~s})=150^{\circ} \mathrm{C}$ matches the expected analytical values directly computed from the thermal coefficient for the Austenitic phase $\left(\alpha\left(150^{\circ} C\right)=9.4 \times 10^{-6}{ }^{\circ} C^{-1}\right)$, both the analytical values $\Delta_{T}$ and the simulated values $\Delta_{T(\operatorname{sim})}=\Delta_{(\operatorname{sim})}-\Delta_{P(\text { sim })}$ for the expansion along each of the axis are quoted to 6 precision digits in table 3 .

The expansion along the $x$-axis and $z$-axis at the time of $1 s$ obtained from the simulation are presented in figure 2 .
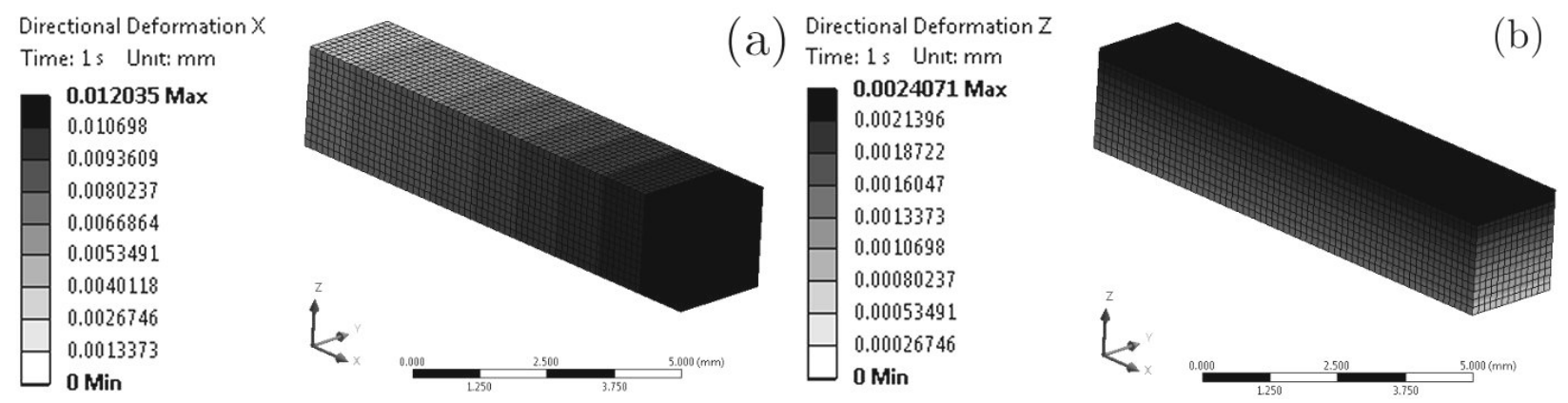

Fig. 2: Deformation along the $x$-axis (a) and $z$-axis (b) at the time of $1 s$ obtained from the simulation for $1 / 4$ of a NiTi bar with dimensions $4 \mathrm{~mm} \times 4 \mathrm{~mm} \times 10 \mathrm{~mm}$. The bar axis is align with the $x$ axis for $y=z=0$.

As for the expansion along the $x$-axis and numerical CTE (4) as functions of the temperature are plotted in figure 3 representing the thermal hysteresis cycle.

The shape memory effect does not play a direct role in the present study, however let us show that it is also properly modeled for the setup just described. Considering the same geometry representing 

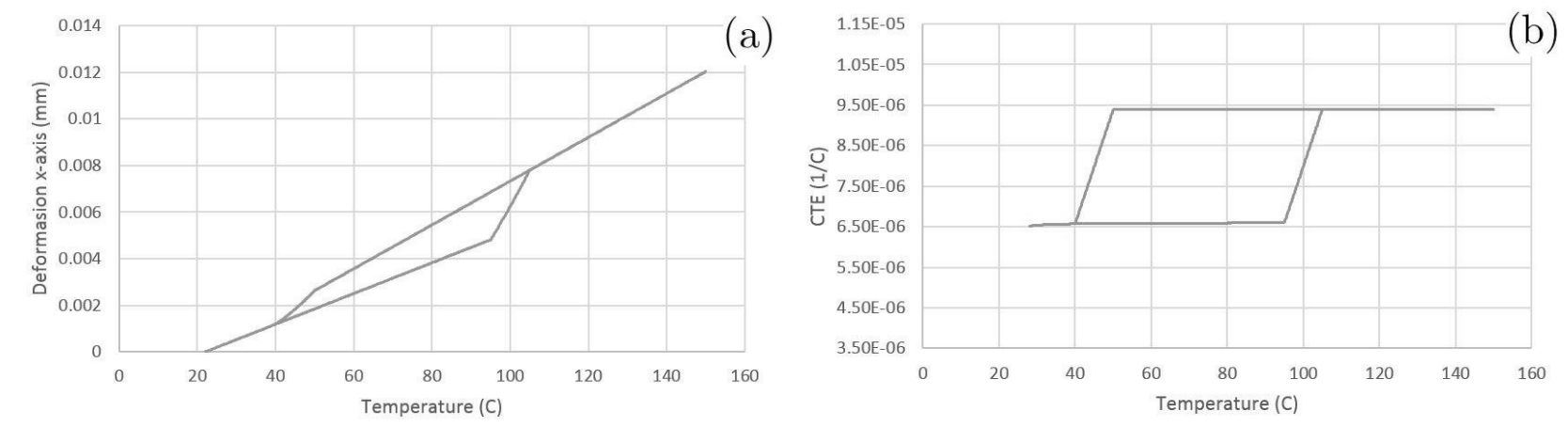

Fig. 3: (a) Deformation along the $x$-axis as a function of temperature. (b) Numerical CTE (4) as a function of Temperature.

a full bar of section $2 \mathrm{~mm} \times 2 \mathrm{~mm}$ and length of $10 \mathrm{~mm}$ with the section at $x=0 \mathrm{~mm}$ fixed and the section at $x=10 \mathrm{~mm}$ moving along the $x$-axis by an amount of $0.76 \mathrm{~mm}$ from the time $t=0.01 \mathrm{~s}$ to the time $t=0.3 \mathrm{~s}$ we obtain a modeled strain of $7.6 \%$ which is enough to exemplify the shape memory effect. Releasing the bar section (at $t=0.3 \mathrm{~s}$ ) we obtain the plastic deformation along the $x$-axis of $0.60403 \mathrm{~mm}$ (the deformation difference $0.15597 \mathrm{~mm}$ corresponds to the elastic strain) and further heating the bar to the temperature of $150^{\circ} \mathrm{C}$ the bar is restored to its previous length at environment temperature of $22^{\circ} \mathrm{C}$. The deformed geometry at time $t=0.37 \mathrm{~s}$ and the restored geometry at $t=0.8 \mathrm{~s}$ is presented in figure 4 . As for the stress hysteresis cycle is plotted in figure 5 for the stress and deviatory stress component $x x$.
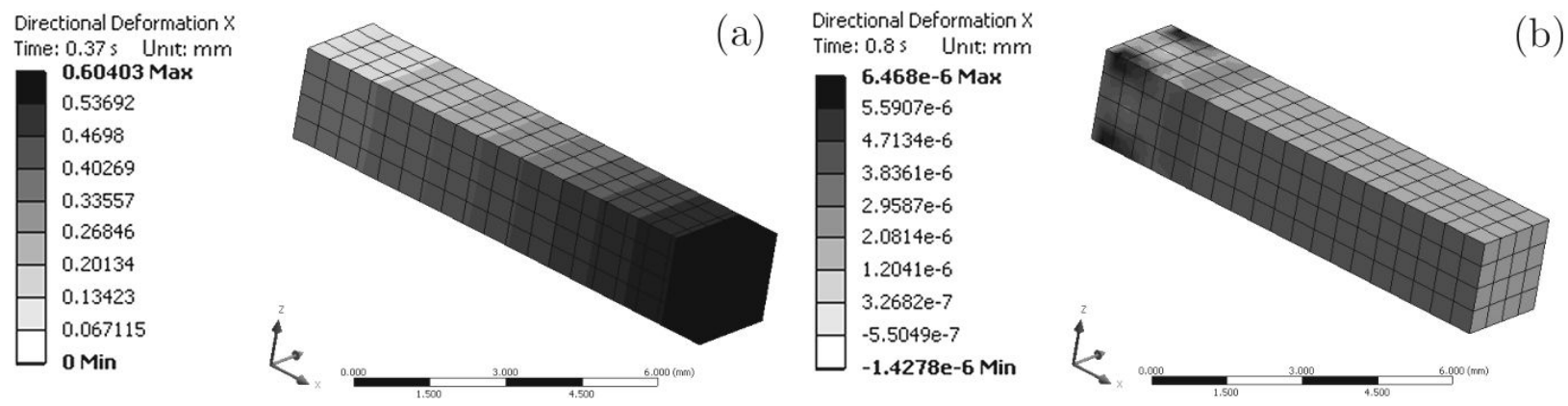

Fig. 4: Exemplification of the shape memory effect: (a) Deformation along the $x$-axis at time $t=0.3 \mathrm{~s}$ corresponding to a strain of $6 \%$ (deformation representation scale is $\times 1$ ). (b) Shape restoration after heating to $150^{\circ} \mathrm{C}$ and cooling to $22^{\circ} \mathrm{C}$ (deformation representation scale is $\times 1$ ). 

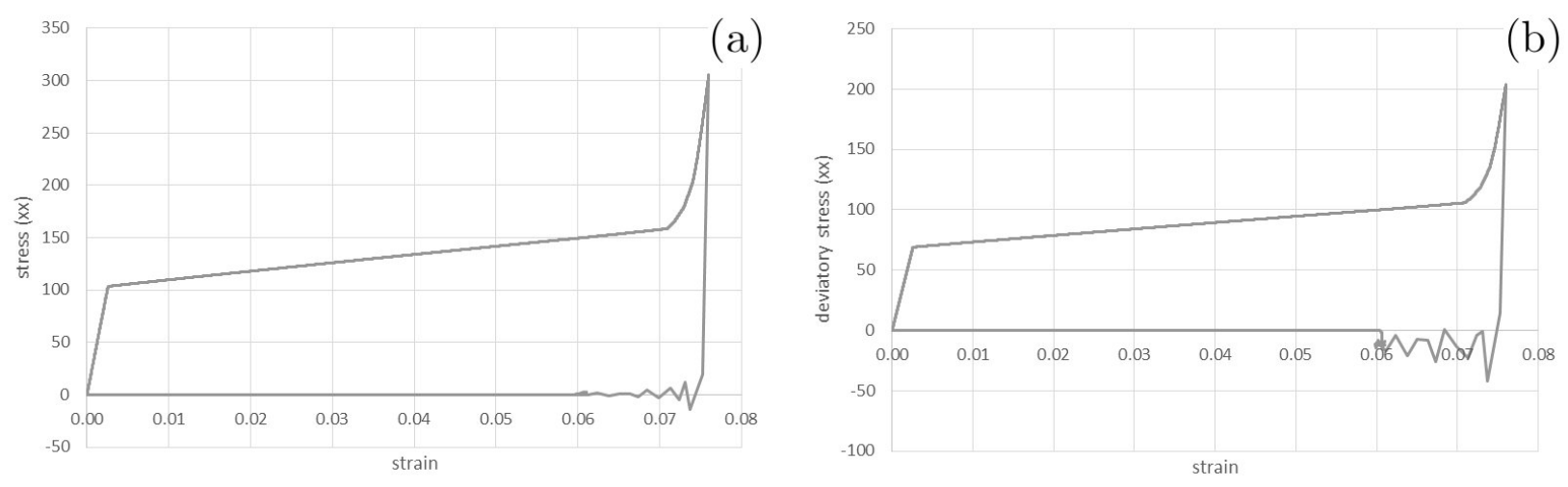

Fig. 5: The full shape memory effect hysteresis cycle including heating and cooling (corresponding to the lower horizontal line from strain of 0.06 to strain of 0.0): (a) Stress component $x x$ plotted as a function of the strain. (b) Deviatory stress component $x x$ plotted as a function of the strain.

\section{Fast Varying Working Conditions}

In the previous section we have considered instantaneous homogeneous heating of the full volume. In real working conditions often the Nitinol parts are subject to fast variation of temperature and pressure. As it takes some time for the bulk temperature to rise to the surface temperature, there is a temperature gradient across the section and the part expansion only matches the surface temperature after some time. This is due to the heat conduction across the part section (from the surface boundary to the bulk) not being instantaneous, it is ruled by the standard heat conduction equations [9]. As an example let us consider the same geometry and a surface temperature profile rising from $T(t=0.1 \mathrm{~s})=22^{\circ} \mathrm{C}$ to $T(t \geq 1 \mathrm{~s})=150^{\circ} \mathrm{C}$ for the 2 external faces of constant $y$ and $z$ such that the simulated geometry is interpreted as a section of a larger part (the remaining faces are, for simulation purposes considered perfectly insulated). The time evolution of the maximum and minimum temperature obtained are plotted in figure 6 , as well as a temperature contour at time $t=1 \mathrm{~s}$.
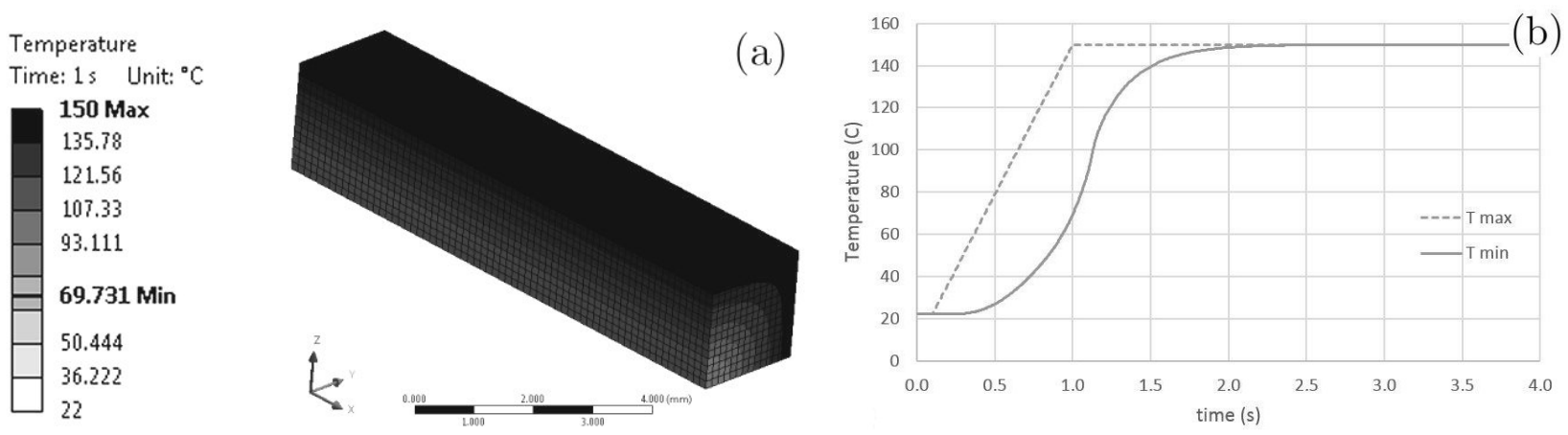

Fig. 6: (a) Temperature contour at $t=1 \mathrm{~s}$. (b) Maximum and minimum temperature as a function of time.

We note that only at time $t \approx 2.5 \mathrm{~s}$ the part is fully homogeneously heated to the temperature $T=150^{\circ} \mathrm{C}$. Also, only at $t \approx 2.5 \mathrm{~s}$ the part expansion will match the expected value for $T=150^{\circ} \mathrm{C}$ while, for instance at $t=1 \mathrm{~s}$, will expand only up to $80 \%$ of that value. We present in figure 7 the expansion along the 3 axis at $t=1 \mathrm{~s}$ and the plot of the external faces expansion $(y, z$-axis deformation) and of the length expansion ( $x$-axis deformation) as a function of time.

We note that, as the temperature rise is not homogeneous, both the Austenitic phase and Martensitic phase will generally be present while the part heats such that the coefficient of thermal expansion 

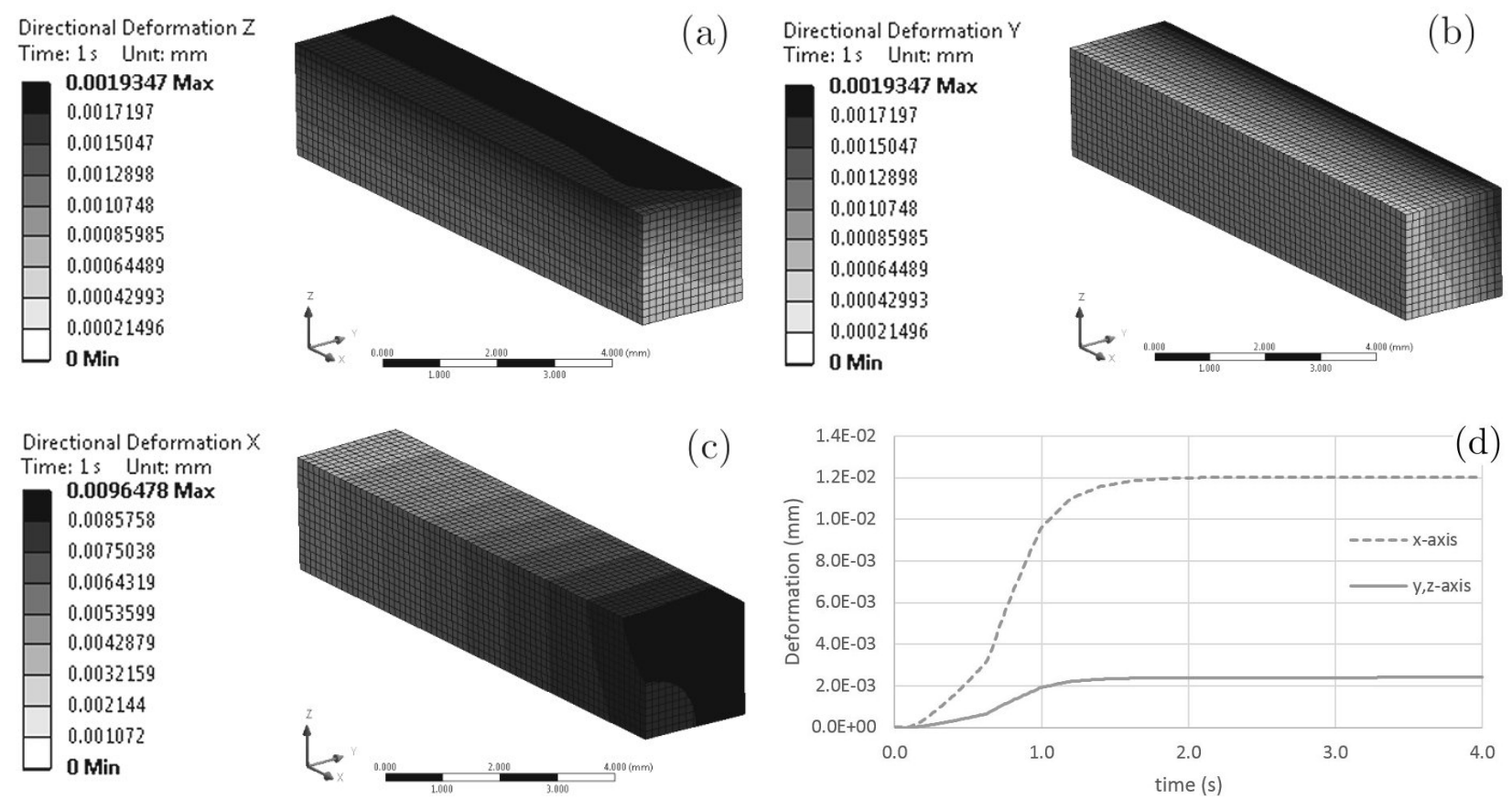

Fig. 7: (a) Deformation along the $z$-axis at $t=1 \mathrm{~s}$. (b) Deformation along the $y$-axis at $t=1 \mathrm{~s}$. (c) Deformation along the $x$-axis at $t=1 s$. (d) Deformations as a function of time.

is varying across the part section. In addition when superficial treatments for the part are being considered the NiTi material properties may also be varying across the section (even for homogeneous heating) such that it is harder to carry an analytical computation for the part expansion.

For larger parts than our simple test geometry the heating time is larger and the temperature gradient becomes more significant. Our simulation is useful for parts intended to work on relatively high temperature environments for short time periods (without preheating of the part) and for which the exact dimensions at working conditions are known. In the next section, as an example, we consider a topological optimization for a simple hollow part also of square section.

\section{Topological Optimization - An Example}

As an example of applicability of the simulation presented in this work let us consider a part of square section with dimensions $10 \mathrm{~mm} \times 10 \mathrm{~mm}$ and undetermined length. For the simulation we are considering a length of $10 \mathrm{~mm}$ and $1 / 4$ of the part with the symmetry axis aligned with the $x$ axis. The part is intended to work for about $t=1.2 \mathrm{~s}$ under a temperature profile that rises from environment temperature $T(t=0.1 s)=22^{\circ} \mathrm{C}$ to $T(t=1 \mathrm{~s})=150^{\circ} \mathrm{C}$ and a pressure profile that rises from $P(t=0.05 s)=0.1 M P a$ to $P(t=0.2 s)=P_{\max }$. We note that the homogeneous heating of the part to the work temperature would correspond to a expansion of $\Delta_{x}=\Delta_{y}=0.00601600 \mathrm{~mm}$ with respect to the part symmetry axis ( $x$-axis), however for the specified working conditions this value will never be reached as the material in the bulk of the part will not have time to reach the external surface temperature.

We are further assuming that the part can be hollow with the inner cavity filled with air which we consider at $t=0 \mathrm{~s}$ to be at a pressure of $0.1 \mathrm{MPa}$ and environment temperature of $22^{\circ} \mathrm{C}$. We will aim at optimizing both the part sectional deformation ( $y, z$-axis) and the material amount (volume) required to manufacture the part. For this purpose the only parameter of the topological optimization will be the wall thickness of the geometry $l_{w} \in[0.5,5] \mathrm{mm}$ where $l_{w}=5 \mathrm{~mm}$ corresponds to a solid part and for analysis parameters we are considering the $y, z$-axis expansion of the part due to both temperature and pressure at the time $t=1.2 \mathrm{~s}$. We consider two distinct cases: 
(i) $P_{\max }=0.1 \mathrm{MPa}$ demanding a maximum working transversal deformation at $t=1.2 \mathrm{~s}$ of $\left|\Delta_{y}\right|=\left|\Delta_{z}\right|<0.005 \mathrm{~mm}$ which corresponds simply to an optimization on the thermal expansion.

(ii) $P_{\max }=80 \mathrm{MPa}$ demanding a maximum working transversal deformation at $t=1.2 \mathrm{~s}$ of $\left|\Delta_{y}\right|=\left|\Delta_{z}\right|<0.02 \mathrm{~mm}$.

For the temperature profile simulation we are considering a setup with the external surfaces heated to the working temperature and the remaining surfaces considered to be perfect insulated (representing the part symmetry and sectioning). As for the structural analysis we are considering a setup with the inner surfaces subject to a pressure of $0.1 \mathrm{MPa}$ and the outer surfaces subject to the pressure profile $P(t)$. Both these setups are represented in figure 8 for a solid and a hollow part.

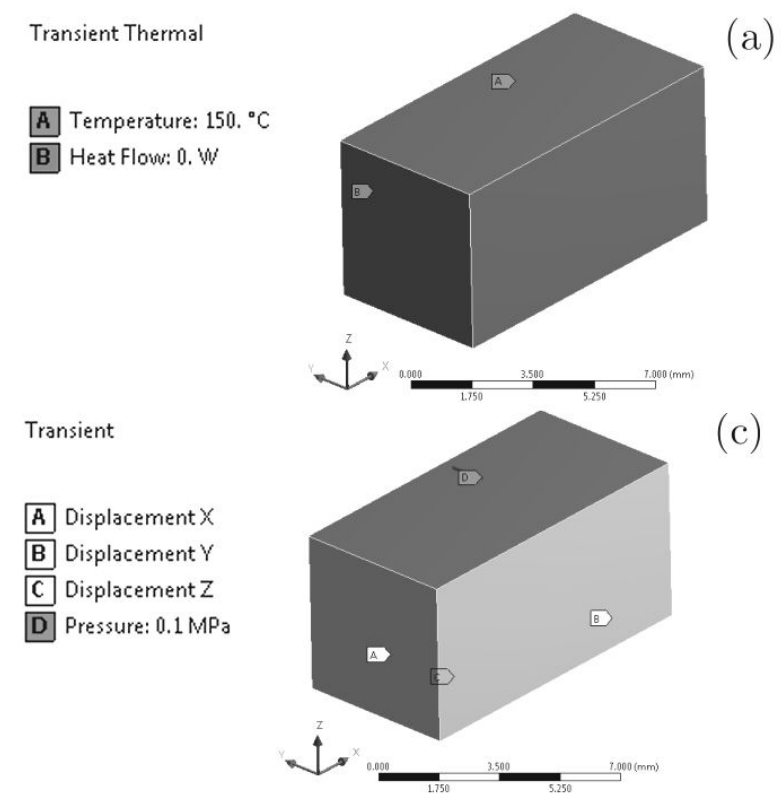

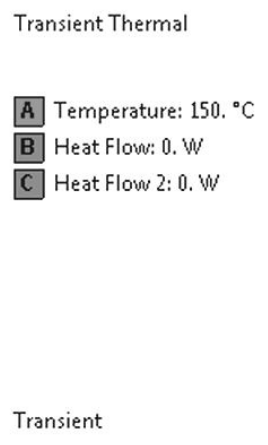
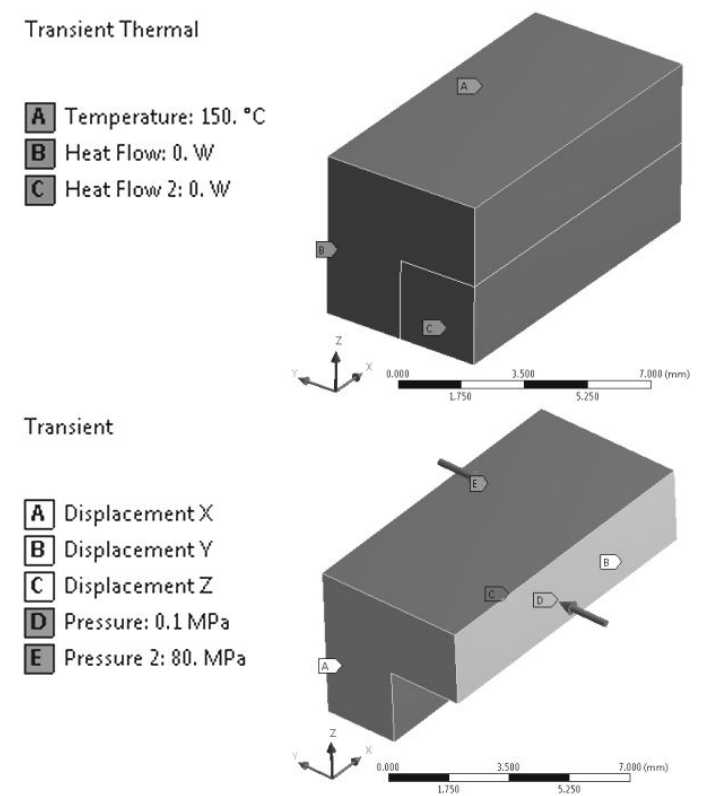

(b)

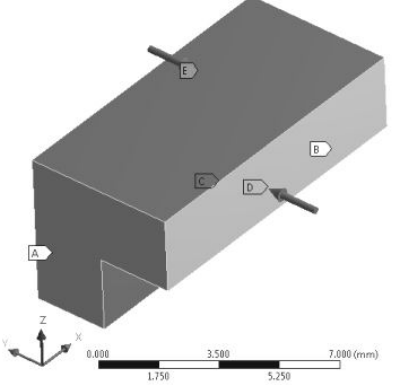

\section{d)}

Fig. 8: (a) Simulation setup for heating of solid part corresponding to the wall thickness of $t_{w}=$ $5 \mathrm{~mm}$. (b) Simulation setup for heating of hollow part with wall thickness $t_{w}=2.5 \mathrm{~mm}$. (c) Simulation setup for thermal structural analysis of solid part $t_{w}=5 \mathrm{~mm}$. (d) Simulation setup for structural thermal and pressure analysis of hollow part with wall thickness $t_{w}=2.5 \mathrm{~mm}$.

An example of the temperature profile for the part at $t=1.2 \mathrm{~s}$ is plotted in figure 9 and for the part deformation along the $z$-axis is plotted in figure 10 .

Running several thermal and structural simulations for distinct values of the wall thickness $t_{w}$ we obtain both the part thermal expansion and deformation under pressure at time $t=1.2 \mathrm{~s}$ as a function of this parameter. We plot the results obtained in figure 11.

The topological optimization process should be iterative such that for the points for which the optimization parameters are near the desired parameter value (aim) further optimization points must be generated (using for example the bisection method). For the cases we are analyzing we obtain for case (i) the value of $t_{w}=1.875 \mathrm{~mm}$ corresponding to a deformation of $\left|\Delta_{x}(t=12 \mathrm{~s})\right|=\mid \Delta_{y}(t=$ $12 s) \mid=0.00492400 \pm 0.00000492 \mathrm{~mm}$ and for the case (ii) the value $t_{w}=2.375 \mathrm{~mm}$ corresponding to a deformation of $\left|\Delta_{x}(t=12 s)\right|=\left|\Delta_{y}(t=12 s)\right|=0.0175020 \pm 0.0001750 \mathrm{~mm}$. We note that we have use as only parameter the part deformation strictly at the time $t=1.2 \mathrm{~s}$, as already mentioned the value of the deformation is not uniform over time and depending on the application the topological optimization may include as additional parameters the deformation at several distinct times. Also generally and depending on the applications additional analysis are often considered such as the part fatigue under the working cycle of the part. 

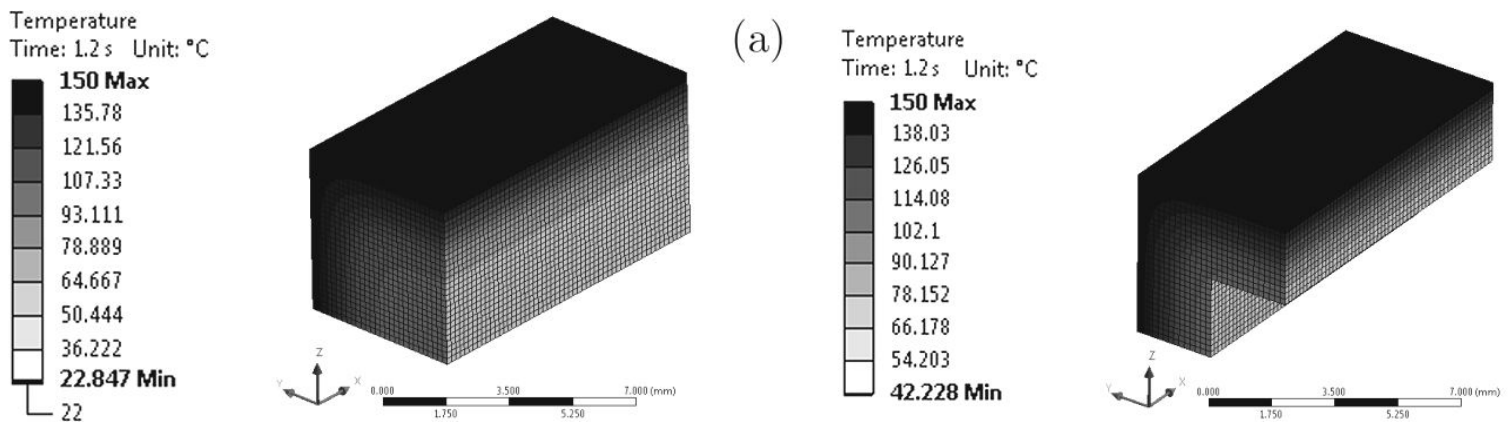

(b)

Fig. 9: Contour plot of the temperature at $t=1.2 \mathrm{~s}$ for the (a) solid part corresponding to the wall thickness of $t_{w}=5 \mathrm{~mm}$ and (b) hollow part with wall thickness $t_{w}=2.5 \mathrm{~mm}$.
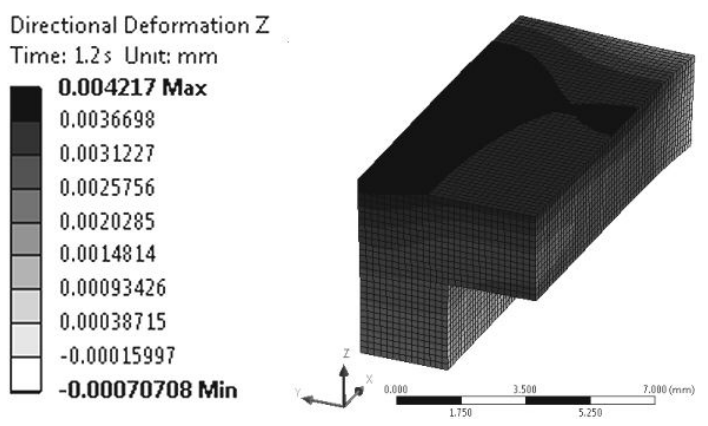

(a)
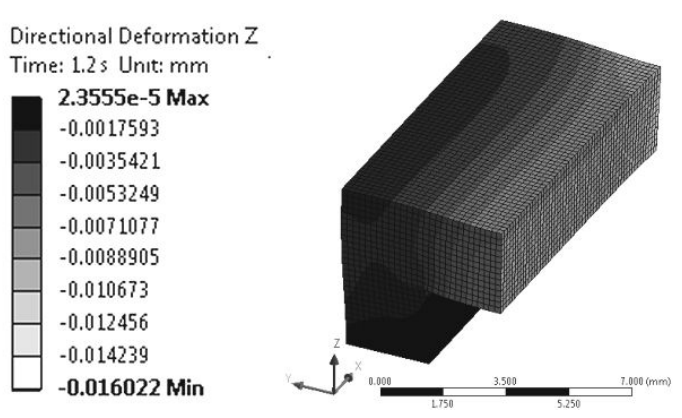

(b)

Fig. 10: Deformation along the $z$-axis at $t=1.2 \mathrm{~s}$ for an hollow part with wall thickness $t_{w}=2.5 \mathrm{~mm}$ for (a) optimization case (i) due to thermal heating (deformation representation scale is $\times 62$ ) and (b) optimization case (ii) due to thermal heating and pressure of $80 M P a$ (deformation representation scale is $\times 29$ ).
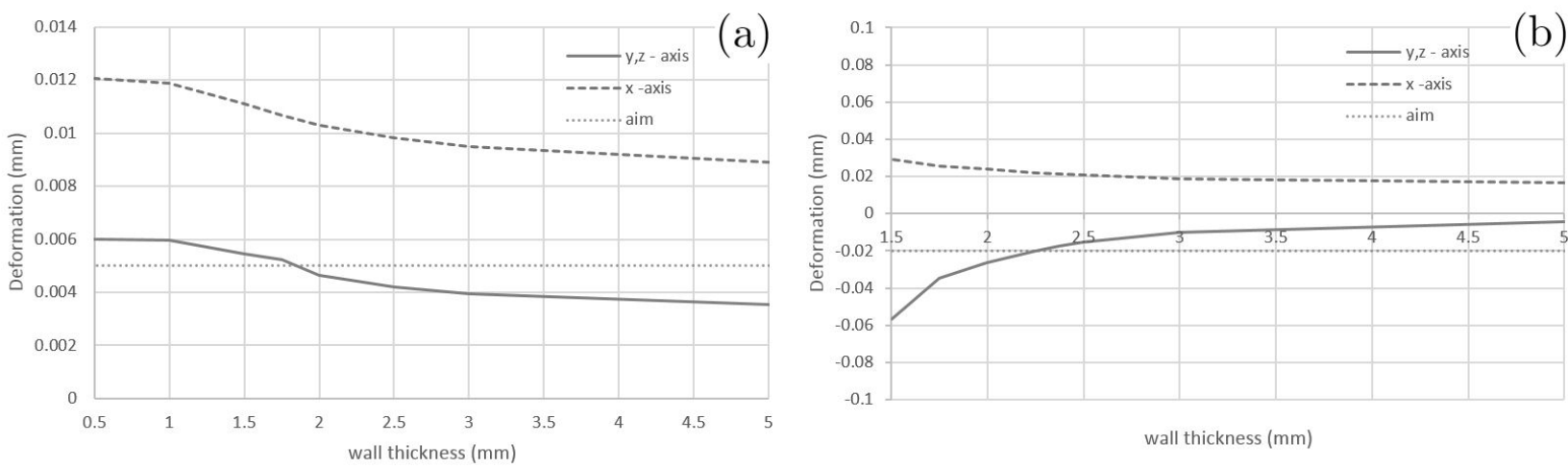

Fig. 11: (a) T. O. Case (i): Deformation due to thermal expansion at $t=1.2 \mathrm{~s}$ as a function of the wall thickness $t_{w}$. Optimal parameter obtained is $t_{w}=1.875 \mathrm{~mm}$. (b) T. O. Case (ii): Deformation due to thermal expansion and external pressure of $80 \mathrm{MPa}$ at $t=1.2 \mathrm{~s}$ as a function of the wall thickness $t_{w}$. Optimal parameter obtained is $t_{w}=2.375 \mathrm{~mm}$. 


\section{Applicability to Direct Digital Manufacturing - Injection Bullet}

As a possible example of applicability of the simulation and topological optimization developed here let us consider one of the bullet geometries discussed in [10] and intended to water assisted injection molding. These bullets aim at hollowing the plastic parts at the molding phase of compactification and the process consists on injecting water in the back of the bullet such that it is propelled forward inside the molded plastic. Often these bullets are, for research purposes, directly manufactured by extrusion molding as described in [10]. For this specific simulation we are considering a bullet made of Nitinol with cylindrical symmetry around the $x$-axis, maximum radius of $9 \mathrm{~mm}$ and length of $23 \mathrm{~mm}$, and for the simulation setup we consider only $1 / 4$ of the bullet geometry as represented in figure 12. Let us further consider the same temperature profile of the previous section rising from the temperature $T(t=0.1 \mathrm{~s})=22^{\circ} \mathrm{C}$ to $T(t=1 \mathrm{~s})=150^{\circ} \mathrm{C}$. To simulate the injected water we consider the pressure profile normal to the back (inside) surface of the bullet rising from $P_{\text {in }}(t=0.05 s)=$ $0.1 \mathrm{MPa}$ to $P_{\text {in }}(t=0.2 \mathrm{~s})=25 \mathrm{MPa}$ and to simulate both the plastic pressure and dynamic friction we consider (respectively) a pressure profile normal to the front (outside) surface of the bullet rising from $P_{\text {out }}(t=0.05 s)=0.1 \mathrm{MPa}$ to $P_{\text {out }}(t=0.2 \mathrm{~s})=16 \mathrm{MPa}$ and a directional pressure profile parallel to the bullet symmetry axis applied to the front (exterior) surface of the bullet rising from $P_{\text {out (dyn) }}(t=0.05 s)=0.0 M P a$ to $P_{\text {out }(\text { dyn })}(t=0.2 s)=5 M P a$ (with direction from the front to the back of the bullet).
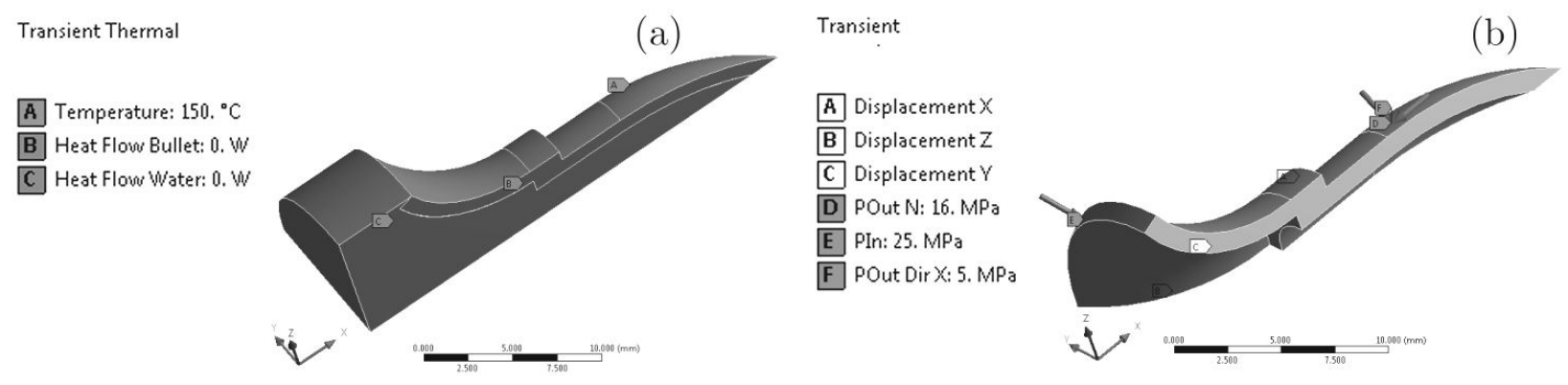

Fig. 12: Simulation setup for $1 / 4$ of the bullet geometry with wall thickness $t_{w}=1 \mathrm{~mm}$ : (a) for thermal heating including the water geometry. (b) for thermal and pressure structural analysis taking in consideration the cylindrical symmetry of the geometry.

For topological optimization of the Nitinol bullet we are considering as only parameter the wall thickness $t_{w} \in[0.25,2.5] \mathrm{mm}$, as in the previous section we are aiming at optimizing the volume maintaining the radial deformation at the time $t=1.2 \mathrm{~s}$ below a critical threshold. Specifically we consider the two distinct cases:

(i) $P_{\text {in }}=P_{\text {out }}=0.1 \mathrm{MPa}$ and $P_{\text {out }(\mathrm{dyn})}=0 \mathrm{MPa}$ demanding a maximum radial deformation over the time period $t \in[0.2,1.2] \mathrm{s}$ of $\left|\Delta_{y}\right|=\left|\Delta_{z}\right|<0.01 \mathrm{~mm}$ which corresponds simply to an optimization on the thermal expansion.

(ii) $P_{\text {in,max }}=25 M P a, P_{\text {out,max }}=16 M P a$ and $P_{\text {out(dyn),max }}=5 \mathrm{MPa}$ demanding a maximum radial deformation over the time period $t \in[0.2,1.2] \mathrm{s}$ of $\left|\Delta_{y}\right|=\left|\Delta_{z}\right|<0.02 \mathrm{~mm}$.

As an example of the simulations results we present in figure 13 the deformation for the bullet geometry with wall thickness $t_{w}=1 \mathrm{~mm}$.

Running the simulations for several wall thickness and employing the bisection method we obtain for the topological optimization (i) $t_{w}=1.00 \mathrm{~mm}$ corresponding to a maximum radial deformation of $\left|\Delta_{y}\right|=\left|\Delta_{z}\right|=0.00984510 \pm 0.00000985 \mathrm{~mm}$ and for the topological optimization (ii) $t_{w}=0.563 \mathrm{~mm}$ corresponding to a maximum radial deformation of $\left|\Delta_{y}\right|=\left|\Delta_{z}\right|=0.0197790 \pm$ $0.0001978 \mathrm{~mm}$. We plot the results obtained for both optimization cases in figure 14 . 


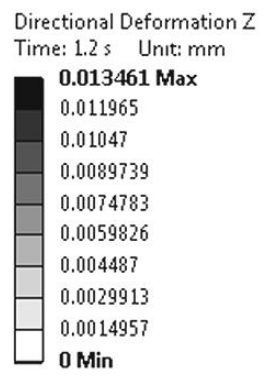

Directional Deformation $X$ Time: $1.2 \mathrm{~s}$ Unit: $\mathrm{mm}$

$0.0098142 \mathrm{Max}$

0.0081376

0.0064611

0.0047845

0.003108

0.0014314

$-0.00024514$

$-0.0019217$

$-0.0035982$

$-0.0052748 \mathrm{Min}$
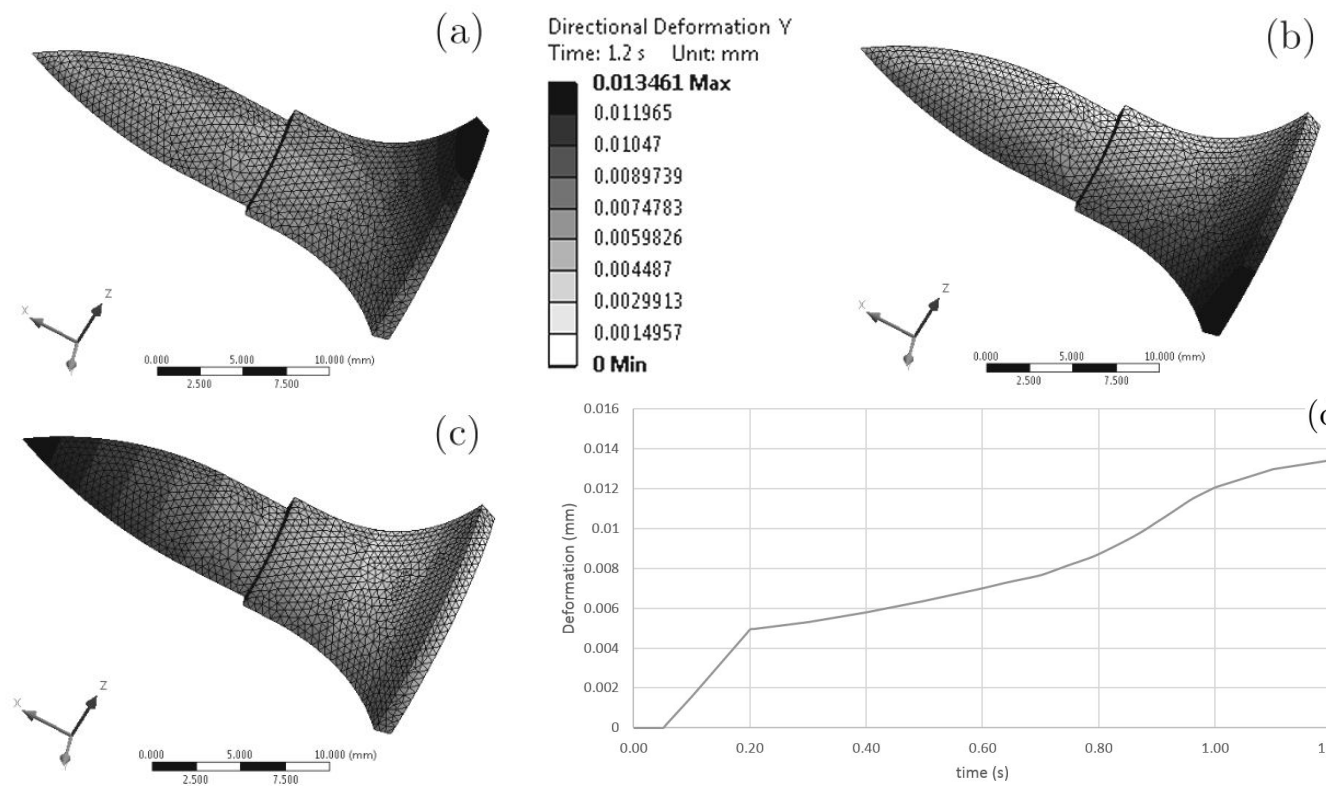

(b)

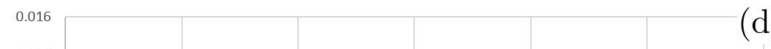

(d)

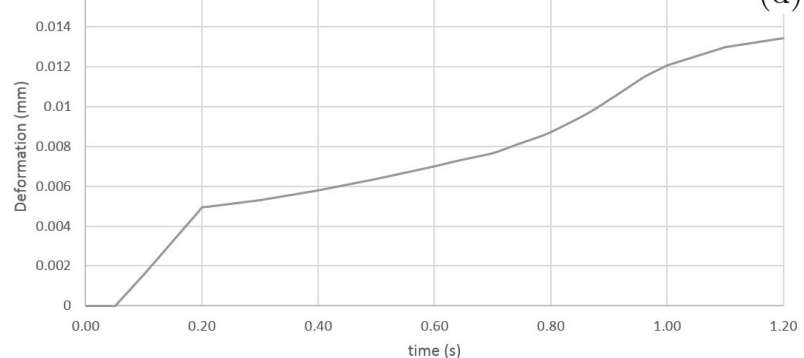

Fig. 13: Deformation for the bullet geometry with $t_{w}=1 \mathrm{~mm}$ under the conditions of optimization case (ii): (a) along the $z$-axis at $t=1.2 \mathrm{~s}$. (b) along the $y$-axis at $t=1.2 \mathrm{~s}$. (c) along the $x$-axis at $t=1.2 s$. (d) maximum radial deformation evolution over time.
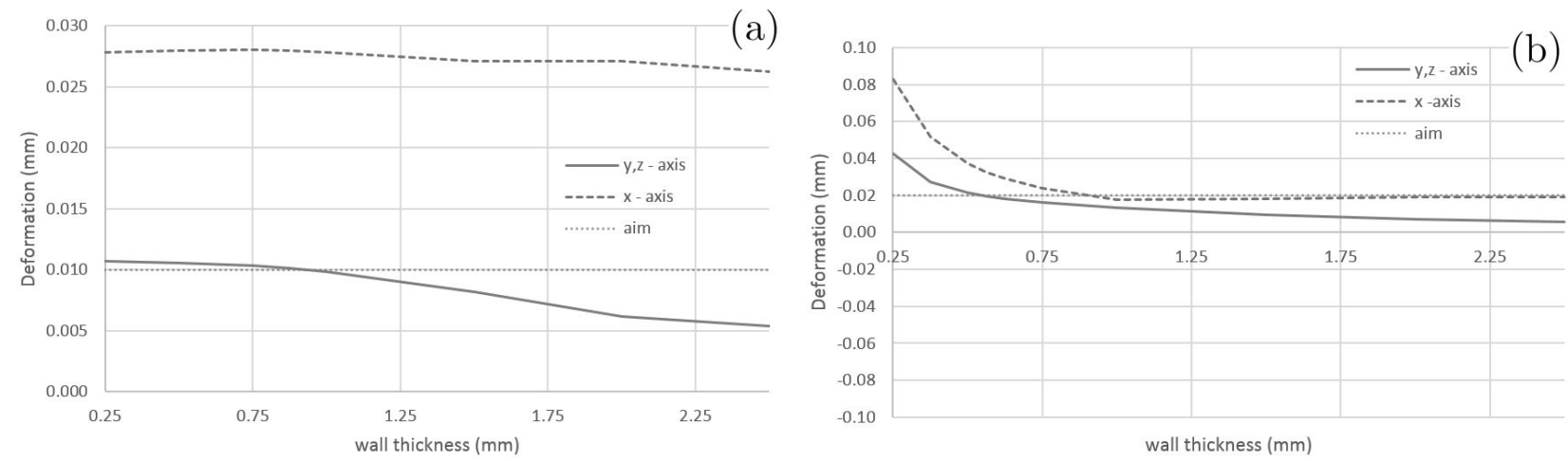

Fig. 14: (a) Bullet T. O. Case (i): Maximum deformation due to thermal expansion as a function of the wall thickness $t_{w}$. Optimal parameter obtained is $t_{w}=1 \mathrm{~mm}$. (b) Bullet T. O. Case (ii): Maximum deformation due to thermal expansion and pressure as a function of the wall thickness $t_{w}$. Optimal parameter obtained is $t_{w}=0.563 \mathrm{~mm}$.

\section{Conclusions}

In this study we have setup a simulation in ANSYS to model the Nitinol thermal expansion reproducing the thermal hysteresis cycle. We have also discussed possible applications for parts modeling under fast varying working conditions, in particular we have presented an explicit topological optimization example for both a square section geometry and a bullet geometry in the context of directly manufactured parts for water assisted injection molding. We recall that as discussed in the introduction both the alloy percentages, the manufacturing process and the treatment affect the Nitinol material properties for both phases, hence either the material to be simulated should already have known properties or the properties must be measured before setting up a simulation that matches the real part. 


\section{Acknowledgements}

This work was supported by the Portuguese Foundation for Science and Technology (FCT) through the Project reference UID/Multi/04044/2013, by the Portuguese Foundation for Science and Technology (FCT) and European Union through the Centro2020 and MATIS (CENTRO-01-0145-FEDER000014 - 3362) and by Agência Nacional de Inovação trough projects WaterSHAPE - Shape Adaptive Projectile for High Performance Water Assisted Injection Moulding Process (Programa-2020FEDER-08/SI/2015 - 3383) and Multi-path.H2O - Ramification Hollow Injection for Water Assisted Moulding (Programa-2020-FEDER-33/SI/2015 - 17684).

\section{References}

[1] The Alloy That Remembers, Time 1968-09-13; W. J. Buehler, J. V. Gilfrich and R. C. Wiley, Effect of LowTemperature Phase Changes on the Mechanical Properties of Alloys near Composition TiNi, Journal of Applied Physics 34 (1963) 1475; F. E. Wang, W. J. Buehler, and S. J. Pickart, Crystal Structure and a Unique "Martensitic" Transition of TiNi, Journal of Applied Physics 36 (1965) 3232.

[2] T. W. Duerig and A. R. Pelton, TiNi Shape Memory Alloys, Materials Properties Handbook Titanium Alloys (1994) 1035-1048.

[3] J. Uchil, K. P. Mohanchandra, K. Ganesh Kumara, K. K. Mahesh and T. P. Murali, Thermal expansion in various phases of Nitinol using TMA, Physica B270 (1999) 289-297.

[4] R. R. Adharapurapu and K. S. Vecchio, Superelasticity in a New BioImplant Material: Ni-rich 55NiTi Alloy, Experimental Mechanics 47 (2007) 365-371.

[5] M. K. Stanford, Thermophysical Properties of 60-NITINOL for Mechanical Component Applications (2012) NASA/TM2012-216056.

[6] N. Wilmoth, Determining the Mechanical Properties of Lattice Block Structures (2013) NASA/CR2013-217880.

[7] A. R. Pelton, J. Dicello and S. Miyazaki, Optimisation of processing and properties of medical grade Nitinol wire, Min. Invas. Ther. \& Allied Technol. 9(1) (2000) 107-118.

[8] L. Petrini, F. Migliavacca, P. Massarotti, S. Schievano, G. Dubini and F. Auricchio, Computational Studies of Shape Memory Alloy Behavior in Biomedical Applications, Transactions of the ASME 127 (2005) 716-725.

[9] ANSYS documentation.

[10] N. Sachin, A. Mateus, P. Carreira, P. Pascoal-Faria and N. Alves, Guided programmable projectiles for Water assisted injection molding, to appear in the proceedings of the 2nd ICDDMAP 2017. 